\title{
DIFERENCIAÇÃO DAS ICNOESPÉCIES E VARIANTES DE Arthrophycus E SUA UTILIZAÇÃO PROBLEMÁTICA EM ICNOESTRATIGRAFIA: O RESULTADO DE HOMOPLASIAS COMPORTAMENTAIS ENTRE ANELÍDEOS E ARTRÓPODES?
}

Trabajo presentado a las XVIII Jornadas de Paleontología y II Congreso Ibérico de Paleontología Universidad de Salamanca

Salamanca, 24-29 de Septiembre de 2002

\author{
Carlos NETO DE CARVALHO ${ }^{1}$, António C. S. \\ FERNANDES ${ }^{2}$ e Leonardo BORGHI ${ }^{3}$
}

\begin{abstract}
${ }^{1}$ Centro de Geologia da Universidade de Lisboa. Bloco C2, $5^{\circ}$ piso, Campo Grande, P-1700 Lisboa, Portugal. Grupo PALEO - Grupo de Paleontologia do Museu Nacional de História Natural. Rua da Escola Politécnica, no 58, P1294 Lisboa CODEX, Portugal. E-mail: praedichnia@hotmail.com.

${ }^{2}$ Departamento de Geologia e Paleontologia - Museu Nacional, Universidade Federal do Rio de Janeiro, Quinta da Boa Vista, São Cristovão, 20942, Rio de Janeiro, Brasil. Departamento de Estratigrafia e Paleontologia, Faculdade de Geologia, Universidade do Estado do Rio de Janeiro. Rua São Francisco Xavier, n 524, Pavilhão João Lyra, $4^{\circ}$ and., sl. 4030, bl. A, Maracanã, 20550013, Rio de Janeiro, Brasil. E-mails: fernande@acd.ufrj.bre fernande@uerj.br. ${ }^{3}$ Departamento de Geologia, Instituto de Geociências, Centro de Ciências Matemáticas e da Natureza, Universidade Federal do Rio de Janeiro. Av. Brigadeiro Trompowsky, s/n, Cidade Universitária, Ilha do Fundão, 21949-900, Rio de Janeiro, Brasil. E-mails: lborghi@igeo.ufrj.bre lborghi@ccard.com.br.
\end{abstract}




\section{INTRODUÇÃO}

Arthrophycus é hoje, irónica e simultaneamente, a designação dada a um dos cinco sub-géneros de Sargassum, uma alga abundante nos mares do Hemisfério Sul, e a uma forma de comportamento preservada no registo estratigráfico (icnofóssil) característica do Paleozóico Inferior, outrora considerada como fósseis de algas sifonáceas. O icnogénero Arthrophycus Hall, 1852 é conhecido em Portugal desde os trabalhos paleontológicos pioneiros de Nery Delgado no Paleozóico Inferior, sob as formas $A$. cfr. harlani (Hall) (Delgado, 1884, 1885, 1888), A. harlani (Costa, 1931), A. sp. (e.g., Cooper e Romano, 1982) e A. alleghaniensis (Harlan, 1831) (Neto de Carvalho et al., 1999). A. harlani foi considerada por James (1893) como sinónimo júnior de A. alleghaniensis.

Até recentemente, este icnogénero era considerado monoicnoespecífico (Fernandes e Borghi, 1996; Fernandes et al., 2000), sendo frequente nas formações siliciclásticas marinhas cambrico-devónicas da Laurentia e Gondwana. Seilacher (2000) subdividiu o icnogénero na icnoespécietipo A. alleghaniensis, em A. linearis e A. lateralis, procurando salientar o seu potencial icnoestratigráfico. Quanto à primeira icnoespécie, considerou-a como indicador bioestratigráfico do Silúrico Inferior (ocorrendo em E.U.A., Argentina e Norte de África). A icnoespécie $A$. linearis foi subdividida nas isubpp. A. linearis protrusiva, indicadora do Ordovícico Superior e observada no Benin e na Jordânia, e A. linearis retrusiva, própria do Ordovícico Inferior (Líbia e Argélia) ao Silúrico Inferior (E.U.A. e Argentina); A. lateralis foi apenas observada no Silúrico Inferior da Líbia (Seilacher op. cit.). Este modelo icnoestratigráfico está a ser utilizado com sucesso nas formações das bacias do Amazonas e Paraná (Brasil). Com efeito, (Fernandes et al., 2001) identificaram preliminarmente A. alleghaniensis no Grupo Trombetas, datado do Llandoveriano (Nogueira et al., 1999) e Formação Vila Maria na Chapada dos Guimarães, no Estado de Mato Grosso, A. linearis no Llandoveriano da Formação Vila Maria no Estado de Goiás (vide Fernandes, 1999) e A. lateralis no Silúrico terminal-Eodevónico da Formação Furnas (vide Fernandes et al., 2000). No entanto, a reavaliação do conteúdo paleoicnológico presente no Ordovícico Inferior português actualmente em curso verificou a ocorrência na Formação do Quartzito Armoricano de A. alleghaniensis e de A. linearis em litofácies argilopsamíticas, em alternâncias centimétricas, do tipo offshore nerítico sob influência tempestítica. Em Portugal, esta formação foi datada do Arenigiano-Oretaniano pela ocorrência de palinomorfos (Paris, 1981), da associação do grupo Cruziana rugosa d'Orbigny, 1842 (Seilacher, 1970) com C. imbricata Seilacher, 1970 (Rebelo e Romano, 1986) e com C. rouaulti Lebesconte, 1883 (Neto de Carvalho et al., 1999), bem como pelas macroassociações (graptólitos e trilobites) que ocorrem na base da forma-

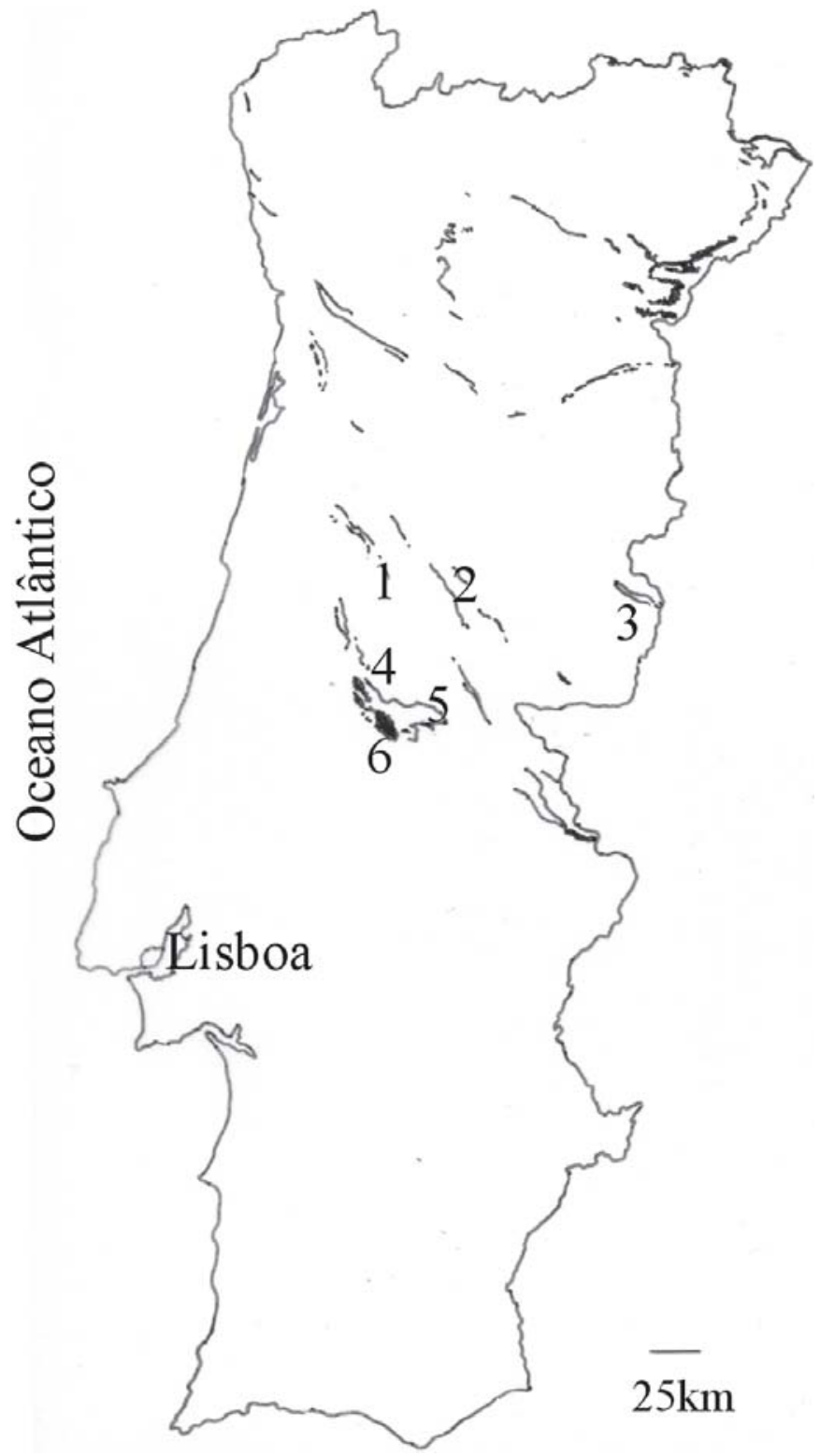

Figura 1. Enquadramento geográfico dos icnofósseis estudados. As manchas escuras salientam a distribuição da Formação do Quartzito Armoricano em Portugal. Os sectores abordados neste trabalho são localizados por números: 1 - proximidades do v.g. Penedo (Penedos de Góis); 2 - caminho da Carvalheira (Vidual de Cima); 3 - garganta do Ponsul (Penha Garcia); 4 corte de estrada E.N. 244, km 22-25 (Amêndoa); 5 Serra de Águas Quentes (Ladeira); 6 - corte de estrada E.N. 2, km 379 (Santiago de Montalegre). The geographical setting of studied ichnofossils. Dark blotches show Armorican Quartzite Formation distribution in Portugal. Sectors approached by this work are located by numbers: 1 - near Penedo summit (Penedos de Gois); 2 - Carvalheira path (Vidual de Cima); 3 - Ponsul gorge (Penha Garcia); 4 - road section E.N. 244, km 22-25 (Amêndoa); 5 Serra de Águas Quentes (Ladeira); 6 - road section E.N. 2, km 379 (Santiago de Montalegre). 
ção xistenta imediatamente sobrejacente (Romano, 1982; Romano et al., 1986; Couto et al., 1997). As formas de Arthrophycus são relativamente comuns nas icnoassociações do Fenniano-Oretaniano (parte superior da Formação do Quartzito Armoricano, Delgado, 1885, Romano, 1991; ocorrência (?) pontual na base da Formação de Colorada, Perdigão, 1965, est. 3, fig. 1, Piçarra, 2000). Não obstante, foram ainda observadas por outros autores abaixo desta sequência, no Tremadociano? do Membro de Sarnelha (McDougall et al., 1987) e, possivelmente, na Formação de Bando dos Santos, datada do Silúrico terminal-Devónico basal (Romão, 2000).

O presente trabalho promove uma análise destas icnoespécies a partir de numerosas amostras observadas e recolhidas no campo (depositadas no Museu Nacional de História Natural) e das colecções de Nery Delgado existentes no Museu do Instituto Geológico e Mineiro (aqui assinaladas com a sigla MGIGM). Os locais de análise e amostrados foram assinalados na Fig. 1. No total, foram medidos o diâmetro, a inclinação e distância entre anelações para 172 exemplares.

\section{ICNOLOGIA SISTEMÁTICA}

\section{Arthrophycus alleghaniensis (Harlan, 1831)}

$$
\text { Figs. 2a-d }
$$

pars 1885 Arthrophycus cfr. Harlani Delgado, 73-75, est. 35, figs. 1-3.

pars 1888 Arthrophycus cfr. Harlani Delgado, 66-70 (parte), est. 9, figs. 1-3.

1980 Arthrophycus sp. indet. Cooper, 374-375, est. 36, fig. 3. 2000 Arthrophycus lateralis Seilacher, 244-245, figs. 5, 6. 2000 Arthrophycus alleghaniensis Seilacher, 243-244, figs. $1,3,4$.

Diagnóstico emendado: galerias do tipo Arthrophycus Hall, 1852 em feixes tridimensionais com estruturas backfill retrusivas ou protrusivas, podendo ramificar em cacho ou curvar tendencialmente num único sentido, a partir de um ponto comum.

Arthrophycus Hall, 1852 type burrows in tridimensional bundles with protrusive or retrusive backfill structures, which may ramify from a single point in a palmate fashion or bend asymmetrically.

Estratigrafia: Câmbrico Inferior (Espanha); Arenigiano-Oretaniano (Portugal, Espanha); Silúrico Inferior-Médio (E.U.A., Argentina, Norte de África); Silúrico basal (Brasil).

Descrição: Os exemplares portugueses classificados como Arthrophycus alleghaniensis são formados por um aglomerado de galerias fasciculares retrusivas dispostas em U ou J (Fig. 2a), com ramificação a partir de um ponto comum menos profun- do, divergentes em leque profundo ou curvando num arco mais ou menos amplo (variante denominada de Arthrophycus lateralis Seilacher, 2000; Figs. 2a-2c). As galerias possuem secção subcircular, com um bandado anelar transverso e regular ao longo do seu comprimento, apresentando 4 anelações $/ \mathrm{cm}$. A base das galerias é aplanada. As anelações transversas são oblíquas $\left(40^{\circ}\right.$ de inclinação média) nas paredes verticais da galeria (backfill lateral), podendo indicar o sentido de deslocamento. Os feixes podem ainda desenvolver-se segundo um modo "teichichnóide" (spreite próximo do vertical; Fig. 2b). Do nosso ponto de vista, A. lateralis parece ser apenas uma variação em arco horizontal dentro do programa de A. alleghaniensis, face a condições de sedimentação/erosão e oxigenação distintas que terão influenciado inclusivamente a posição no nível de ocupação e o timing de ocupação do substrato. As restantes icnotaxobases de $A$. alleghaniensis podem ser diagnosticadas em A. lateralis. Esta icnoespécie pode ainda apresentar o mesmo padrão divagante e com entrecruzamento de estruturas, de A. linearis (Fig. 2d). O preenchimento passivo das galerias difere da matriz siltítica quase homogénea pela sua composição quartzo-arenítica grosseira heterogranular.

É comum A. alleghaniensis ocorrer em relação estreita com o grupo Cruziana rugosa em número acessório (e.g., Serra da Amêndoa) ou, mais raramente, em associações monoicnoespecíficas com índices de bioturbação no plano de camada iguais ou superiores a 4 (sensu Miller e Smail, 1997), de que são exemplos o corte da EN2, ao km 379 (Vila de Rei) e o corte do caminho da Carvalheira (Vidual de Cima).

\section{Arthrophycus linearis Seilacher, 2000}

Figs. 3a-d

pars 1885 Arthrophycus cfr. Harlani Delgado, 73-75, ests. 23, 36.

pars 1888 Arthrophycus cfr. Harlani Delgado, 66-70, est. 10.

2000 Arthrophycus linearis Seilacher, 241-243, figs. 1, 2.

Diagnóstico: Galerias do tipo Arthrophycus não ramificadas ou raramente fazendo-o, rectilíneas ou ligeiramente curvas, paralelas ao plano de camada. A sua secção transversal mostra-se protrusiva (lamelas do backfill com a convexidade voltada para cima) ou retrusiva (lamelas com a convexidade voltada para baixo); Seilacher (2000).

Arthrophycus burrows with no or few side branches, running straight or smoothly curving, along bedding planes. Cross section of spreite is either protrusive (backfill lamella convex-up) or retrusive (lamella convex-down); Seilacher (2000).

Estratigrafia: A. linearis: Silúrico Inferior (Brasil); A. linearis tipo protrusiva: Arenigiano-Oretaniano (Portugal); Ordovícico Superior (Benim, Jordânia). A. linearis tipo retrusiva: Ordovícico Inferior (Líbia, Argélia); Silúrico Inferior (E. U. A., Argentina).

Descrição: As Figs. 3a e 3b mostram a laje $(1,48 \mathrm{~m}$ x 0,83 m) coberta com Arthrophycus linearis tipo protrusiva proveniente dos Penedos de Góis, recuperada e analisada, mas não figura- 

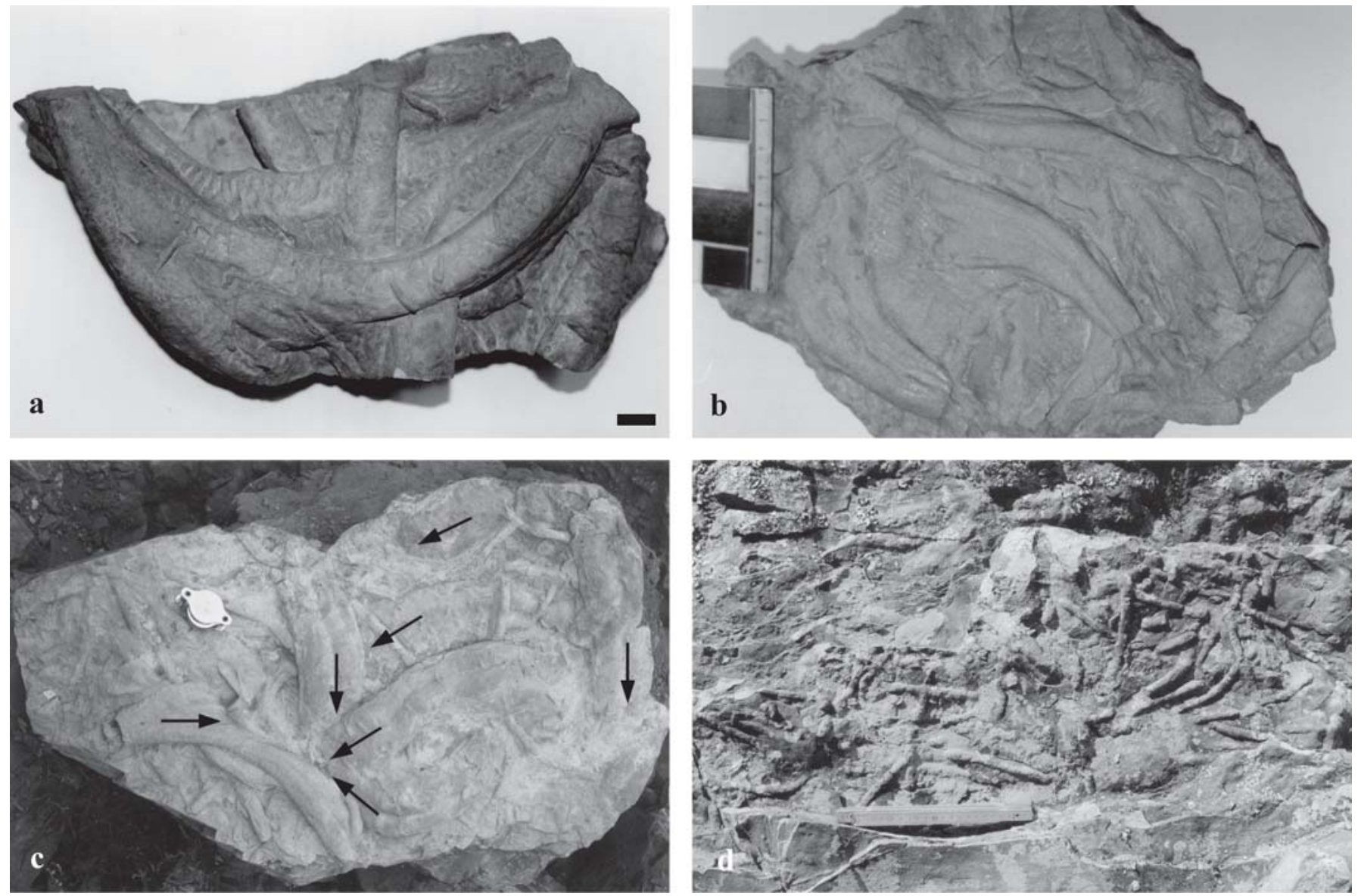

Figura 2. Arthrophycus alleghaniensis do Ordovícico de Portugal. a - MGIGM13184, Arthrophycus alleghaniensis tipo lateralis (Fenniano-Oretaniano, Sinclinal de Amêndoa-Carvoeiro - nas proximidades do sector 6); escala = 10 mm; Delgado (1888), p. 69, est. 9, fig. 1. b-Arthrophycus alleghaniensis tipo lateralis (Fenniano-Oretaniano, Sinclinal de Amêndoa-Carvoeiro - sector 4), colecção privada de Carlos Farinha, referência OMC00825; escala $=10 \mathrm{~cm}$. c - Arthrophycus alleghaniensis tipo lateralis, em nível de ocupação mais profundo, a cortar um "ponto de encontro" de Cruziana goldfussi (Rouault, 1850); as setas indicam o sentido de deslocamento (Fenniano-Oretaniano, Sinclinal de Amêndoa-Carvoeiro - sector 5); escala $=40 \mathrm{~mm}$. d - Arthrophycus alleghaniensis (Fenniano, Sinclinal de Penha Garcia - sector 3); escala = $20 \mathrm{~cm}$. Ordovician Arthrophycus alleghaniensis from Portugal. a - MGIGM13184, Arthrophycus alleghaniensis, lateralis type (Fennian-Oretanian, Amêndoa-Carvoeiro Syncline - not far from sector 6); scale = $10 \mathrm{~mm}$; Delgado (1888), p. 69, pl. 9, fig. 1. $\boldsymbol{b}$ - Arthrophycus alleghaniensis, lateralis type (Fennian-Oretanian, Amêndoa-Carvoeiro Syncline - sector 4), this sample belongs to the private Carlos Farinha collection with the reference OMC00825; scale $=10 \mathrm{~cm}$. $\mathrm{c}$ - Arthrophycus alleghaniensis, lateralis type, in a deeper tier, cross-cutting Cruziana goldfussi (Rouault, 1850) "meeting point", arrows indicate ploughing directions assumed by Cruziana producers (Fennian-Oretanian, Amêndoa-Carvoeiro Syncline - sector 5); scale $=40 \mathrm{~mm} . \boldsymbol{d}$ - Arthrophycus alleghaniensis (Fennian, Penha Garcia Syncline - sector 3); scale = $20 \mathrm{~cm}$.

Figura 3. Arthrophycus linearis no Ordovícico de Portugal. a - MGIGM13107, Arthrophycus linearis (Fenniano, Sinclinal de Buçaco - sector 1); escala = $10 \mathrm{~cm}$. b - MGIGM13107, A. linearis, o mesmo nível estratigráfico do que o da Figura 3a (Fenniano, Sinclinal de Buçaco - sector 1); escala $=10 \mathrm{~cm}$. c - MGIGM4933, A. linearis com anelações em chévron, desligadas junto do eixo axial e com desfasamentos que só podem ser explicados por movimentos articulados de apêndices (Fenniano, Sinclinal de Buçaco - sector 1); escala = $10 \mathrm{~mm}$. d - Variante em profundidade do circling behaviour comum a Cruziana semiplicata Salter, 1854 e ao grupo Cruziana rugosa, observado em A. linearis (Fenniano-Oretaniano, Sinclinal de Amêndoa-Carvoeiro, não longe do sector 4); escala = 10 mm; Delgado (1888), p. 68, est. 10.

Ordovician Arthrophycus linearis from Portugal. a - MGIGM13107. Arthrophycus linearis (Fennian, Buçaco Syncline sector 1); scale $=10 \mathrm{~cm} . \boldsymbol{b}-$ MGIGM13107, A. linearis, the same stratigraphic horizon as in Figure 3a (Fennian, Buçaco Syncline - sector 1); scale $=10 \mathrm{~cm} . \mathrm{c}$-MGIGM4933, A. linearis with chévron-like constrictions disconnected near the sagittal axis, in a non-bilateral symmetric arrangement, which can only be explained by incised movements of articulated appendages (Fennian, Buçaco Syncline - sector 1); scale $=10 \mathrm{~mm} . \mathrm{d}$-Depth modification of the circling behaviour common to Cruziana semiplicata Salter, 1854 and to the rugosa-group, now showed in, and genetically related with, A. linearis (Fennian-Oretanian, Amêndoa-Carvoeiro Syncline - not far from sector 4); scale =10mm; Delgado (1888), p. 68, pl. 10 . 

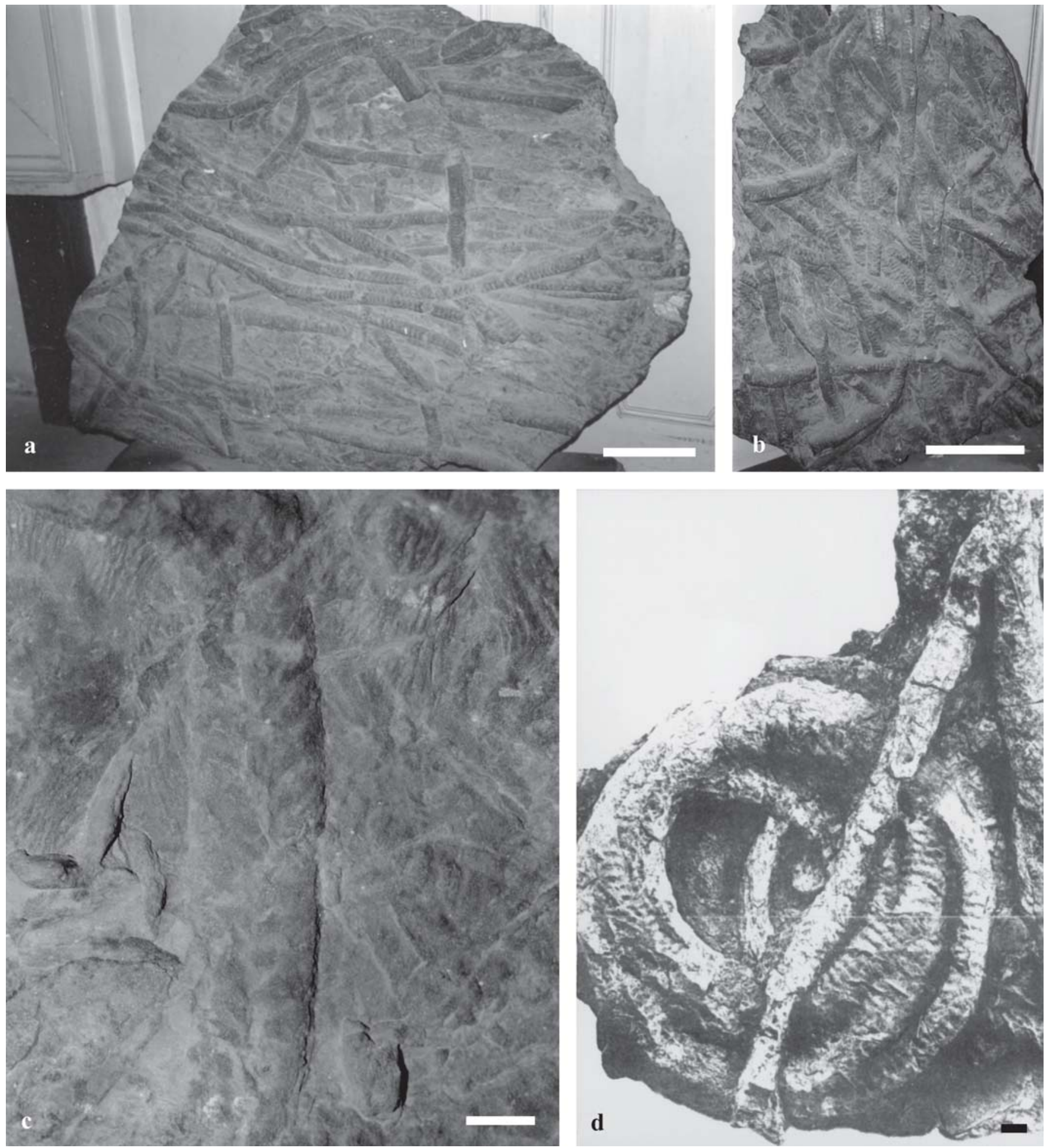

da, em Delgado (1885). As ichnosubpp. definidas por Seilacher com base na orientação das lamelas de backfill são aqui consideradas tipos ou variantes morfo-etológicas definidas localmente pelo regime de sedimentação/erosão vigente e não como morfotipos com icnotaxobases descriminativas. Correspondem a galerias dispostas horizontalmente ao longo do plano de estratificação (até 1,2 m de extensão) e não ramificadas, justapostas, por vezes entrecruzadas (sintomático de um posicionamento intrastratal), rectilíneas ou pouco sinuosas. O seu diâme- tro é anormalmente elevado para o icnogénero (até $25 \mathrm{~mm}$ ); apresentam uma secção transversal sub-quadrada a elíptica, anelações transversas muito regulares (cerca de 2 lamelas $/ \mathrm{cm}$ ) e uma depressão média longitudinal. As anelações transversas nas formas mais bem preservadas apresentam, por vezes, uma quebra ao nível da depressão central e uma disposição que deixa de ser transversa para tomar uma forma em chévron (marcas de apêndices; Fig. 3c). Neste, mas igualmente em outros exemplos, estas marcas em chévron mostram-se desfasadas, o 
que parece indicar um movimento aductor-abductor (com período de retracção) dos apêndices, por vezes não bilateralmente síncrono. Uma boa parte das estruturas paralelas observadas nas figuras $3 \mathrm{a}$ e $3 \mathrm{~b}$ têm uma largura constante, o que permite inferir uma biocenose produtora dimensionalmente homogénea ou extensa bioturbação (Índice de Bioturbação no plano de camada $=5$ sensu Miller e Smail, 1997) realizada por um único indivíduo, revolvendo o sedimento em duas direcções do espaço, fazendo apenas variar o sentido de locomoção (mantendo-se sub-paralelo à estrutura previamente produzida num modo rectilíneo) e a profundidade no nível de ocupação. As formas de A. linearis adoptam modos de comportamento, como sobreposições em mudança de nível de ocupação, entrecruzamentos curvilíneos (Fig. 3b) e "circling behavior" (Fig. 3d), muito semelhantes àqueles verificados para o grupo Cruziana rugosa em sequências quartzo-pelíticas homólogas de Portugal (e.g., Penha Garcia).

\section{OS POSSÍVEIS PRODUTORES DE Arthrophycus}

Quanto aos produtores de Arthrophycus, e no caso de A. alleghaniensis, a presença de anelações transversas com relação ao plano axial, ao longo das galerias em U, entre as quais, em espécimes bem preservados, se pode observar finos pregueamentos paralelos (cf. Delgado, 1885), para além da presença de um backfilling lateral, testemunham a actuação fodinichnia, com movimentação peristáltica, de um organismo vermiforme celomado sedimentívoro cuja cutícula de revestimento apresenta um fino enrugamento funcionalmente eficaz para facilitar o processo de escavação (implicando o movimento de sedimento ao longo do animal, Seilacher, 2000). Em A. linearis, as evidências do efeito de bulldozing recto e extenso, sem ramificar, bilobado, com transporte de sedimento para o eixo axial através da articulação de movimentos de apêndices, assim como de circling behavior (com componente de deslocação vertical; também observado em Hall, 1852, vol. II, est. 2, Fig. 1C) de raio elevado e com inclinação na área de curvatura (características também observadas em Cruziana d'Orbigny, 1842 na parte superior da Formação do Quartzito Armoricano), apontam para um produtor endobentónico depositívoro, metamerizado e esclerotizado (artrópode, trilobite?). Já em 1885, o ilustre geólogo e arqueólogo português, Nery Delgado, notara a estreita relação genética existente entre Cruziana e Arthrophycus (Delgado, 1885: 72). As populações das duas icnoespécies de Arthrophycus na Formação do Quartzito Armoricano, em Portugal, mostram-se heterogéneas ao nível do diâmetro médio das galerias, para um grau de significância de $95 \%$ (probabilidade associada ao teste T-Student, com uma distribuição unicaudal, de $0.032 ; \mathrm{n}_{\text {A. linearis }}=69, \mathrm{n}_{\text {A. alleghaniensis }}=$ 103). Da mesma forma, a comparação entre as medidas dos diâmetros para as duas icnoespécies de Arthrophycus (Fig. 4) sugere produtores dimensionalmente não correlacioná- veis $(\mathrm{r}=0.21)$. A atribuição de Arthrophycus a mining behaviour de artrópodes tem sido levantada recentemente por diversos autores sem que, todavia, haja qualquer tipo de argumentação de suporte (Fernandes, 2000; Mayoral, 2000; Rindsberg, 2001). A invariância dimensional ao longo dos dois morfotipos acima descritos indica que estes não terão sido produzidos no decurso de uma vida.

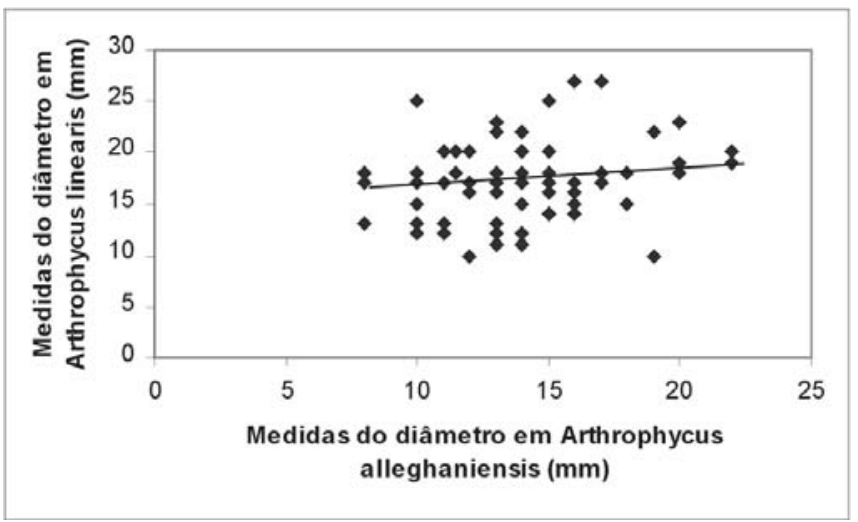

Figura 4. Gráfico de correlação entre as medidas do diâmetro de Arthrophycus linearis versus Arthrophycus alleghaniensis. O coeficiente de correlação, dado pelo declive da recta de regressão, é $\mathrm{r}=0.21$.

Correlation plot between diameter measures of Arthrophycus linearis versus Arthrophycus alleghaniensis. Correlation coefficient, given by the regression line slope, is $r=0.21$.

\section{CONSEQUÊNCIAS DAS OBSERVAÇÕES E INTERPRETAÇÕES FORMULADAS COM BASE NAS ICNOESPÉCIES DE Arthrophycus DO ORDOVÍCICO INFERIOR DE PORTUGAL}

Os dados acima descritos demonstram que, embora a utilização das icnoespécies de Arthrophycus em correlações cronológicas e estratigráficas regionais esteja a dar frutos no Brasil, os exemplos expostos e as definições problemáticas das novas icnoespécies e relativas variantes preservacionais (icnotaxobases não diagnósticas) poderão colocar em causa a utilização bioestratigráfica das formas de Arthrophycus em outros sectores da Gondwana. Como paradigma, Baldwin (1975) e Liñan (1984) observaram em Espanha a ocorrência de A. alleghaniensis no Arenigiano da Formación Barrios e no Câmbrico basal da Formación Torrearboles, respectivamente. Estas icnoespécies e suas variantes não parecem corresponder a morfotipos característicos de uma determinada idade, mostrando uma evolução no sentido da complexificação etológica (Seilacher, 2000), mas a programas comportamentais intrínsecos, controlados pelas condições físicas do meio (taxa de sedimentação/erosão, variação do potencial redox), de grupos de 
organismos (vermes e artrópodes) que tiveram o seu desenvolvimento etológico marcado por convergência evolutiva e restrito ao Paleozóico Inferior.

\section{AGRADECIMENTOS}

Os autores desejam expressar a sua gratidão ao Instituto Geológico e Mineiro, nas pessoas do Professor Dr. Miguel Ramalho e do Dr. José Brandão, pela permissão para a análise das colecções de Nery Delgado e demais facilidades; a Carlos Farinha, pela cedência de uma amostra de Arthrophycus alleghaniensis var. lateralis da sua colecção privada (contacto: farinha.c@netcabo.pt).

\section{BIBLIOGRAFIA}

Baldwin, C. T. 1975. The stratigraphy and facies association of trace fossils in some Cambrian and Ordovician rocks in north-western Spain. Trace Fossils 2 (Coord. P. T. Crimes and J. D. Harper). Geological Journal Special Issue, 9, 9-40.

Cooper, A. H. 1980. The Stratigraphy and Palaeontology of the Ordovician to Devonian rocks of the area North of Dornes (near Figueiró dos Vinhos), Central Portugal. Ph. D. Thesis, University of Sheffield, 1-378 (inédito).

Cooper, A. H. and Romano, M. 1982. The Lower Ordovician stratigraphy of the Dornes-Figueiró dos Vinhos area, Central Portugal, with descriptions of Merostomichnites ichnosp. and Rosselia socialis, two previously unrecorded trace fossils. Comunicações dos Serviços Geológicos de Portugal, 68, 73-82.

Costa, J. C. S. 1931. O Paleozóico Português. Síntese e crítica. Tese de Doutoramento (ed. Autor), Faculdade de Ciências da Universidade do Porto, 1-141.

Couto, H., Piçarra, J. M. y Gutiérrez-Marco, J. C. 1997. El Paleozóico del Anticlinal de Valongo (Portugal). In: XIII Jornadas de Paleontologia "fósiles de Galicia" y V Reunión International Proyecto 351 PICG "Paleozoico Inferior del Noroeste de Gondwana”, A Coruna, 1997, Libro de Resúmenes y Excursiones (Coord. A. Grandal d'Anglade, J. C. Gutiérrez-Marco y L. Santos Fidalgo). Sociedad Española de Paleontología, Madrid, 270-290.

Delgado, J. F. N. 1884. Note sur les échantillons de Bilobites envoyés à l'exposition géographique de Toulouse. Societé de Histoire Naturelle de Toulouse, Bulletin., 18, 126-131.

Delgado, J. F. N. 1885. Terrenos paleozóicos de Portugal: Estudo sobre os Bilobites e outros fósseis das quartzites da base do Systema Silurico de Portugal. Memória da Secção de Trabalhos Geológicos de Portugal, Lisboa, 1113.

Delgado, J. F. N. 1888. Terrenos paleozóicos de Portugal: Estudo sobre os Bilobites e outros fósseis das quartzites da base do Systema Silurico de Portugal (Supplemento). Commissão dos Trabalhos Geológicos de Portugal, Lisboa, 1-75.
Fernandes, A. C. S. 1999. Conteúdo icnológico das formações do Ordoviciano-Devoniano da Bacia do Paraná, Brasil. Boletim do Museu Nacional, nova série, Geologia, 46, 1-12.

Fernandes, A. C. S. 2000. A paleoicnofauna brasileira de artrópodes: estado atual de seu conhecimento. In: I Simpósio Brasileiro de Paleoartropodologia (Coord. R. G. M. Neto). Ribeirão Preto, 108-109.

Fernandes, A. C. S. e Borghi, L. 1996. Comentários sobre o icnogénero Arthrophycus Hall, 1852 e seu registro nas bacias sedimentares brasileiras. Anais do Simpósio Sul Americano do Siluro-Devoniano, Ponta Grossa, 131-139.

Fernandes, A. C. S., Borghi, L. e Moreira, M. I. C. 2000. Sobre a ocorrência do icnogénero Arthrophycus Hall, 1852 na Formação Furnas (Bacia do Paraná). Boletim do Museu Nacional, nova série, Geologia, 52, 1-14.

Fernandes, A. C. S., Borghi, L. e Neto de Carvalho, C. 2001. As icnoespécies de Arthrophycus Hall, 1852 e suas ocorrências no Siluriano-Devoniano das bacias do Amazonas e Paraná. XVII Congresso Brasileiro de Paleontologia, Rio Branco (Acre), 25.

Hall, J. 1852. Paleontology of New York. Vol. 2, Albany, 1362.

James, J. F. 1893. Remarks on the genus Arthrophycus Hall. Journal of Cincinnati Museum of Natural History, 16, 8286.

Liñan, E. 1984. Los icnofósiles de la Formación Torrearboles (¿Precámbrico?-Cámbrico Inferior) en los alrededores de Fuente de Cantos, Badajoz. Cuadernos do Laboratorio Xeológico de Laxe, 8, 47-72.

Mayoral, E. 2000. Pistas fósiles de artrópodos. IX Congresso Ibérico de Entomología. Sociedad Entomológica Aragonesa, Zaragoza, 42-43.

McDougall, N., Brenchley, P. J., Rebelo, J. A. and Romano, M. 1987. Fans and fan deltas - precursors to the Armorican Quartzite (Ordovician) in western Iberia. Geological Magazine, 124, 347-359.

Miller, M. and Smail, S. E. 1997. A Semiquantitative Field Method for Evaluating Bioturbation on Bedding Planes. Palaios, 12, 391-396.

Neto de Carvalho, C., Ramos, J. e Cachão, M. 1999. A "Bicha Pintada" (Vila de Rei, Portugal): uma história de Património (bio)conturbado. Comunicações do I Seminário sobre Património Geológico, Instituto Geológico e Mineiro, Lisboa.

Nogueira, A. C. R., Truckenbrodt, W. e Soares, E. A. A. 1999. O icnogênero Arthrophycus de depósitos sublitorâneos da Formação Nhamundá (Siluriano Inferior) da Bacia do Amazonas, região de Presidente Figueiredo. Revista Brasileira de Geociências, 29, 135-140.

Paris, F. 1981. Les chitinozoaires dans le Paléozoïque du sudouest de l'Europe. Cadre géologique-Étude systématiqueBiostratigraphie). Mémoire de la Société Géologique et Minéralogique de Bretagne, 26, 1-412.

Perdigão, J. C. 1965. Sobre a descoberta de Cruziana e Vexillum (=Daedalus) na colina de Cuncos (Mourão). Comunicações dos Serviços Geológicos de Portugal, 48, 161-167. 
Piçarra, J. M. 2000. Estudo estratigráfico do Sector de Estremoz-Barrancos, Zona de Ossa-Morena, Portugal. Vol. II - Bioestratigrafia do intervalo Ordovícico-Devónico Inferior. Tese de Doutoramento (inédito), Universidade de Évora, 1-173.

Rebelo, J. A. and Romano, M. 1986. A contribution to the lithostratigraphy and palaeontology of the Lower Palaeozoic rocks of the Moncorvo region, Northeast Portugal. Comunicações dos Serviços Geológicos de Portugal, 72, 45-58.

Rindsberg, A. K. 2001. Arthrophycus and the problem of compound trace fossils. North American Paleontological Convention, Berkeley, California.

Romano, M. 1982. The Ordovician Biostratigraphy of Portugal - a review with new data and re-appraisal. Geological Journal, 17, 89-110.

Romano, M. 1991. Lower to middle Ordovician trace fossils from the Central Iberian Zone of Portugal and Spain. In: Advances in Ordovician Geology (Coord. C. R. Barnes and S. H. Williams). Geological Survey of Canada, Paper 90-9, 191-204.

Romano, M., Brenchley, P. J. and McDougall, N. D. 1986. New information concerning the age of the beds immediately overlying the Armorican Quartzite in Central Portugal. Geobios, 19, 421-433.

Romão, J. M. C. 2000. Estudo Tectono-Estratigráfico de um segmento do bordo SW da Zona Centro-Ibérica, e as suas relações com a Zona Ossa-Morena. Tese de Doutoramento (inédito), Faculdade de Ciências da Universidade de Lisboa, 1-323.

Seilacher, A. 1970. Cruziana stratigraphy of non-fossiliferous palaeozoic sandstones. In: Trace Fossils (Coord. P. T. Crimes and J. C. Harper). Geological Journal Special Issue, 3, 447-476.

Seilacher, A. 2000. Ordovician and Silurian Arthrophycid Ichnostratigraphy. In: Geological Exploration in Murzuq Basin (Coord. M. A. Sola and D. Worsley). Elsevier Science B. V., 237-258.

Manuscrito recibido: 19 de diciembre, 2002 Manuscrito aceptado: 11 de julio, 2003 